SLAC-PUB-9351

August 2002

\title{
Stability Analysis of Longitudinal Beam Dynamics Using Non-Canonical Hamiltonian Methods and Energy Principles*
}

\author{
M. Venturini \\ Stanford Linear Accelerator Center, Stanford University, Stanford, CA 94309
}

\begin{abstract}
In the presence of RF focusing and a purely inductive impedance bunch equilibria in the form of Haïssinski distributions - when they exist - are linearly stable. This is the case whether the potential well distortion associated with the impedance causes bunch lengthening or shortening. We provide a general proof of this fact using Hamiltonian methods and energy principles. In the presence of bunch shortening our analysis indicates that there is a critical current for linear stability. However, this threshold is identical to the critical current defining the condition for the very existence of a Haïssinski equilibrium.
\end{abstract}

Published in PRST-AB

*Work supported by Department of Energy contract DE-AC03-76SF00515. 


\section{Introduction}

The study of linear stability of longitudinal beam dynamics in accelerators or storage rings is usually done by normal mode analysis. While rigorous and well established in reference to coasting beams the method is not totally satisfactory when applied to bunched beams.

For coasting beams the problem of linear stability is analogous to that of plasma waves in a homogeneous medium, a complete solution of which was first provided by Landau [1] - the only difference being in the form of the interaction potential. Landau's method is set as an initial value problem involving use of the Fourier (or Laplace) transform on the linearized Vlasov equation but, as later shown by van Kampen and Case [2, 3], the procedure is equivalent to determining the eigenmodes of the system.

The basic complication in treating bunched beams is the coupling between the azimuthal modes that are very naturally introduced to represent the distribution function in phase space. As a consequence, Landau's technique in its original form cannot be applied. Instead, one introduces an additional expansion in radial modes and tries to cast the resulting linearized Vlasov equation in the form of a finite-dimension eigenvalue problem - upon suitable truncation of the expansion [4]. There are various difficulties with this procedure. On the one hand the linearized equation has the form of a singular integral equation - the singularity being where the coherent frequencies equal the single particle frequencies - and its discretization is not well grounded mathematically [5], leaving the convergence with respect to the order of truncation dubious at least. On the other hand, even if normal modes can be determined exactly their completeness is usually difficult or impossible to prove - thus excluding from consideration possible perturbations that cannot be represented as superposition of normal modes.

Because of these limitations it would be of great interest to be able to study the stability problem using methods different from mode decomposition. Natural candidates are the energy principles and Hamiltonian methods that have successfully been employed in a number of problems of plasma physics, astrophysics, and galactic dynamics [6, 7, 8, 9, 10, 11]. When they apply these methods can be very powerful and allow for full characterization of linear and possibly even non-linear stability. Unfortunately, we have to temper the reader's enthusiasm and add that in the study of longitudinal beam dynamics they only apply if the machine impedance is purely inductive. Although quite peculiar this is nevertheless a sufficiently important case to deserve our attention. Purely inductive impedances are often used in theoretical models, including those describing longitudinal space-charge effects [14].

We will discuss two different but closely related approaches. In both cases demonstration of (linear) stability is reduced to proving that a certain functional defined over the space of distribution functions representing deviations from equilibrium is positive definite. These techniques differ in the way the functional is constructed starting from the ascertained existence of a suitable invariant.

The first method $[6,7,8]$ tries to identify the desired invariant by exploiting the Hamiltonian nature of the system. One assumes the point of view that the beam distribution function is itself a dynamical variable in an infinite dimensional Hamiltonian (although not necessarily canonical) field theory. Upon suitable definition of a Hamiltonian functional and 
Lie-Poisson brackets, equivalence is established between the Vlasov equation and the equations of motion for this field theory. The sought invariant is then identified as the effective Hamiltonian generating the linearized equations of motion.

The second method $[9,10,11]$ uses the analogy between the linearized Vlasov equation and that of a harmonic oscillator to identify an invariant quantity analogous to the energy. The analogy is not immediate and only becomes apparent after re-expressing the linearized Vlasov equation as a second order equation in time. This can be done by using a decomposition of the distribution function in even and odd components with respect to its dependence on momentum.

By applying these methods we will be able to show that in the presence of a purely inductive impedance bunch equilibria in the form of Haïssinski distributions are always linearly stable - when they exist. We recall that the potential well distortion associated with a purely inductive impedance can cause either bunch lengthening or shortening - depending on the algebraic sign of the impedance, machine lattice, and beam energy. In the presence of bunch shortening it is possible to determine a critical value for linear stability. However, we will also show that for currents above this threshold a Haïssinski equilibrium does not even exist implying that the linear theory does not apply. The collective forces are so overwhelming that no stationary distribution can be put together.

The rest of the paper is organized as follows. After stating the problem and establishing notation (Sec. 2), we will introduce the two methods separately in Sec. 3 and 4. A discussion of the stability results will be presented in Sec. 5 and the Conclusions will follow.

\section{Vlasov Equation}

Longitudinal beam dynamics in a storage ring is the result of interaction with RF cavities providing longitudinal focusing, coupling with wake fields and - if the current intensity is particularly high - space charge. All these effects are Hamiltonian in nature. In this paper non-Hamiltonian effects due to synchrotron radiation will be neglected except for the purpose of defining physically meaningful equilibria (at least for high-energy electrons).

Following the notation used in $[4,13]$ we work with the normalized variables $q=z / \sigma_{z}$ and $p=-\left(E-E_{0}\right) / \sigma_{E}$, where $z$ is the distance from the synchronous particle, $E$ is the particle energy, and $E_{0}$ the beam design energy. For bunched beams the scaling factors $\sigma_{z}$ and $\sigma_{E}$ are conveniently chosen to obey $\sigma_{E} / \sigma_{z}=E_{0} \nu_{s} /|\eta| R$, where $\nu_{s}$ is the synchrotron oscillation tune, $\eta$ the slippage factor, and $R$ the ring average radius. The above relationship holds if - for instance $-\sigma_{z}$ and $\sigma_{E}$ are taken to be the rms longitudinal size and energy spread of a gaussian bunch (in the limit of vanishing current). In passing, we recall that in the relativistic limit the synchrotron tune $\nu_{s}$ depends on the RF wavelength $\lambda_{r f}$ and peak voltage $V_{r f}$ as $\nu_{s}^{2}=|\eta| e V_{r f} R / E_{0} \lambda_{r f}$, where $e$ is the particle charge.

A beam is customarily described by a distribution function $f(q, p)$ in phase space obeying the Vlasov equation

$$
\frac{\partial f}{\partial t}=[H, f]
$$

where $[.,$.$] are the Poisson brackets and H$ is the 'single-particle' Hamiltonian. Assuming the 
linear approximation for the $\mathrm{RF}$ force, in the canonical coordinates $q$ and $p$ the Hamiltonian $H$ reads:

$$
H=\frac{1}{2} p^{2}+\frac{1}{2} q^{2}+U_{f}(q)
$$

provided that we interpret the independent time-like variable $t$ as $t=s \nu_{s} / R$, where $s$ is the orbit arclength (in which case $\Delta t=2 \pi$ corresponds to one period of synchrotron oscillations). The beam-distribution dependent potential $U_{f}$ describes the action of the wake-field forces. The effect of the wake force is usually modelled in terms of the wake function $W\left(q-q^{\prime}\right)$. Specifically, $W\left(q-q^{\prime}\right) / 2 \pi R$ has the meaning of the longitudinal electric field per unit charge acting on a test particle in $q$ due to a point charge in $q^{\prime}$. We have

$$
U_{f}(q)=I \int_{-\infty}^{q} d q^{\prime} \int_{-\infty}^{\infty} d q^{\prime \prime} W\left(q^{\prime}-q^{\prime \prime}\right) \rho\left(q^{\prime \prime}\right)
$$

where $\rho(q)=\int f(q, p) d p$ is the normalized longitudinal charge density and the parameter $I=\operatorname{sgn}(\eta) N e^{2} / 2 \pi \nu_{s} \sigma_{E}$ (with the meaning of a normalized current) is proportional to the number of particles $N$ in the bunch. Here, we shall be concerned with the specific case of a purely inductive impedance [14] $Z(\omega)=-i \omega L$. We will refer to this impedance as purely inductive whether the parameter $L$ is positive or negative although when $L<0$ one often speaks of purely capacitive impedance [14]. The latter provides a model for longitudinal space-charge effects $[14,15]$.

The wake-function for a purely inductive impedance is the Fourier transform $W(q)=$ $\int d \omega \exp \left(-i \omega \sigma_{z} q / c\right) Z(\omega) / 2 \pi=-\left(c^{2} / \sigma_{z}^{2}\right) \hat{\delta}^{\prime}(q)$ where $\hat{\delta}$ is the Dirac $\delta$-function (here ' denotes derivation with respect to $q)$. With this choice of wake-function the Vlasov equation (1) takes the form:

$$
\frac{\partial f}{\partial t}-\frac{\partial f}{\partial p}\left(q+I_{L} \frac{d}{d q} \int_{-\infty}^{\infty} f\left(q, p^{\prime}\right) d p^{\prime}\right)+p \frac{\partial f}{\partial q}=0
$$

where $I_{L}=I c^{2} / \sigma_{z}^{2}$. Equation (4) admits an infinite number of equilibria: any arbitrary function of the Hamiltonian is, in fact, a stationary solution (i.e. $\partial f_{0} / \partial t=0$ ). However, we restrict ourselves to Haïssinski distributions [14], which are of the form $f_{0}=\hat{f}_{0} \exp \left(-H_{0}\right)$, with $\hat{f}_{0}$ being a normalization constant and $H_{0}$ the Hamiltonian evaluated at $f=f_{0}$, or equivalently

$$
f_{0}=\frac{e^{-p^{2} / 2}}{\sqrt{2 \pi}} \rho_{0}(q)
$$

where $\rho(q)$ is solution of the Haïssinski equation

$$
\frac{d \rho_{0}}{d q}\left(1+I_{L} \rho_{0}\right)=-q \rho_{0}
$$

with normalization $\int \rho(q) d q=1$. We recall that Haïssinski distributions are physical equilibria that are realized in the presence of radiation effects. In the limit $I_{L} \rightarrow 0$ a Haïssinski distribution becomes a gaussian. Solutions corresponding to $I_{L}>0$ (e.g. machine with positive momentum compaction above transition and $L>0$ ) and $I_{L}<0$ display bunch lengthening and shortening respectively (see Fig. 1). We will comment more on the $I_{L}<0$ case later in Sec. 5. 


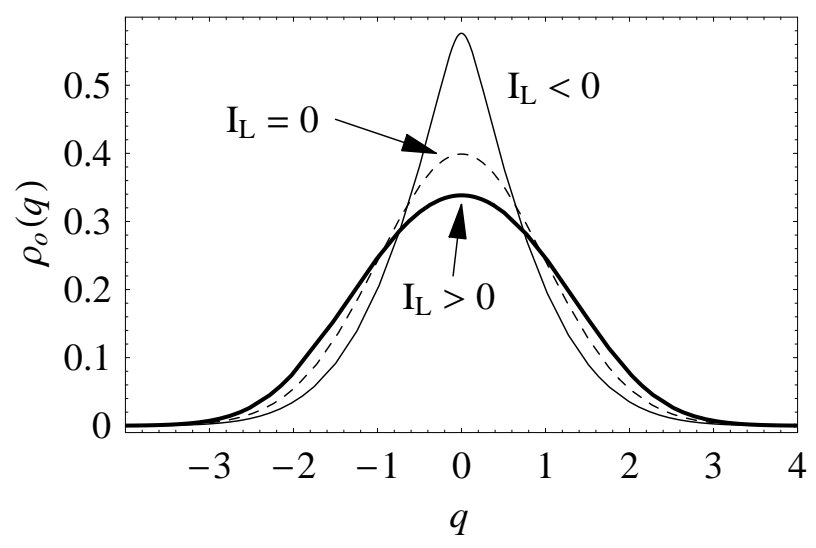

Figure 1: Examples of charge density profiles $\rho_{0}(q)$ of Haïssinski distributions for $I_{L}>0$ (displaying bunch lengthening), $I_{L}<0$ (displaying bunch shortening) and $I_{L}=0$ (gaussian distribution).

The problem we are concerned with is whether Haïssinski equilibria are stable under small perturbations obeying the linearized Vlasov equation. As usual one sets $f=f_{0}+f_{1}$, where $f_{1}$ is a small perturbation and $H=H_{0}+H_{1}$ (where $H_{0}$ and $H_{1}$ are the portions of the Hamiltonian that depend on $f_{0}$ and $f_{1}$ respectively) so that the linearized Vlasov equation reads:

$$
\frac{\partial f_{1}}{\partial t}=\left[H_{0}, f_{1}\right]+\left[H_{1}, f_{0}\right]
$$

with

$$
\begin{aligned}
& {\left[H_{1}, f_{0}\right]=I_{L} \frac{\partial f_{0}}{\partial p} \frac{d}{d q} \int_{-\infty}^{\infty} d p^{\prime} f_{1}\left(q, p^{\prime}\right) \equiv \mathcal{K} f_{1}} \\
& {\left[H_{0}, f_{1}\right]=\left(q+I_{L} \frac{d \rho_{0}}{d q}\right) \frac{\partial f_{1}}{\partial p}-p \frac{\partial f_{1}}{\partial q} \equiv \mathcal{D} f_{1}}
\end{aligned}
$$

The last equalities serve as definitions of the operators $\mathcal{D}$ and $\mathcal{K}$, which will be used later.

The analysis presented in the following will apply to bunched as well to unbunched beams. It is useful then to mention how our notation changes for coasting beams. First, in scaling the canonical variable $z$ it is convenient to set the scaling factor $\sigma_{z}$ equal to the machine average radius $\sigma_{z}=R$ so that $q=z / R$ has the meaning of an angle. Next, by defining the 'time' as $t=s|\eta| \sigma_{E} / E_{0} R$ the Hamiltonian becomes formally the same as in Eq. (2) with the term $q^{2} / 2$ dropped and with the definition of $I$ given by $I=e^{2} N_{c} E_{0} / 2 \pi \sigma_{E}^{2} \eta$, where $N_{c}$ is the total number of circulating particles. The Haïssinski distribution is the same as in Eq. (5) but with constant $\rho_{0}(q)=1 / 2 \pi$. 


\section{Hamiltonian Method}

\subsection{The Non-Canonical Infinite-Dimensional Formalism}

It was first pointed out by Morrison [6] that the Vlasov-Maxwell equations can be viewed in the framework of infinite-dimensional Hamiltonian systems. Here we apply this perspective to the Vlasov equation describing charged particle beams. This entails interpreting a beam distribution function as a 'point' in an infinite dimensional 'phase space'.

The key ingredient in a Hamiltonian system is the existence of a bilinear operator such that the set of dynamical variables together with this operator is a Lie-algebra. We recall that by definition dynamical variables are functions (or functionals - in the infinite dimensional case) with domain in phase space. For finite-dimension canonical Hamiltonian systems such an operator is represented by the familiar Poisson brackets $[\cdot, \cdot]$. The Hamiltonian is characterized as the generator of the time evolution of any dynamical variable including the canonical variables themselves

$$
q^{\prime}=[q, H], \quad p^{\prime}=[p, H] .
$$

As we move to infinite-dimensional and not necessarily canonical systems we want to maintain the notion that a Hamiltonian still generates the equations of motion in conjunction with a more general linear operator - the Lie-Poisson brackets - with all the properties (antisymmetry, Jacobi identity) that one demands in order to have a Lie-algebra.

The challenge is to find suitable definitions of a Hamiltonian $\mathcal{H}$ and Lie-Poisson brackets $\{\cdot, \cdot\}$ so that the equation of motion for this infinite dimensional theory

$$
\frac{d}{d t} \mathcal{F}[f]=\{\mathcal{F}[f], \mathcal{H}[f]\}
$$

is equivalent to the Vlasov equation (1). We emphasize that (11) is a functional equation, the Hamiltonian $\mathcal{H}$ itself is a functional and the Lie-Poisson brackets define an operation between functionals.

Quite naturally, the choice for the Hamiltoninan $\mathcal{H}$ proposed by Morrison is the mean energy of the system. In our case the single-particle Hamiltonian $H$ is

$$
H(q, p ; t)=\frac{1}{2} p^{2}+\frac{1}{2} q^{2}+\frac{I_{L}}{2} \int_{-\infty}^{\infty} d p^{\prime} f\left(q, p^{\prime}\right)
$$

so that for $\mathcal{H}$ we have

$$
\mathcal{H}[f]=\int d q d p f(q, p)\left(\frac{1}{2} p^{2}+\frac{1}{2} q^{2}+\frac{I_{L}}{2} \int_{-\infty}^{\infty} d p^{\prime} f\left(q, p^{\prime}\right)\right) .
$$

The factor $1 / 2$ before the last integral on the RHS comes from avoiding double counting of particle interactions. In turn, the Lie-Poisson brackets are defined through

$$
\{\mathcal{A}, \mathcal{B}\}[f]=\iint d q d p f\left[\frac{\delta \mathcal{A}}{\delta f}, \frac{\delta \mathcal{B}}{\delta f},\right],
$$


where $\delta \cdot / \delta f$ is the functional derivative and $[\cdot, \cdot]$ are the usual Poisson-brackets. This definition is consistent because functional derivatives are functions in $q$ and $p$ and the Poissonbrackets operate on functions. We recall that the functional derivative $\delta \mathcal{A} / \delta f$ of a functional $\mathcal{A}$ is defined as the kernel of the integral expression that identifies the first variation of that functional

$$
\delta \mathcal{A}[f ; \delta f]=\iint \frac{\delta \mathcal{A}}{\delta f} \delta f d q d p .
$$

In turn, first variations can be calculated using the familiar prescription ${ }^{\dagger}$

$$
\delta \mathcal{A}[f ; \delta f]=\left.\frac{d}{d t} \mathcal{A}[f+t \delta f]\right|_{t=0} .
$$

It can be verified [6] that the brackets $\{\cdot, \cdot\}$ are bilinear and are legitimate Lie-Poisson brackets fulfilling the antisymmetry condition and Jacobi identity:

$$
\begin{aligned}
& \{\mathcal{A}, \mathcal{B}\}=-\{\mathcal{B}, \mathcal{A}\} \\
& \{\mathcal{A},\{\mathcal{B}, \mathcal{C}\}\}+\{\mathcal{B},\{\mathcal{C}, \mathcal{A}\}\}+\{\mathcal{C},\{\mathcal{A}, \mathcal{B}\}\}=0
\end{aligned}
$$

Equipped with the machinery of the calculus of variations one is ready to show that equation (11) is, in fact, the Vlasov equation in disguise. To this end we first need to identify those functionals $\mathcal{F}$ that can be related to beam distributions. We choose $\mathcal{F}=\mathcal{F}_{q, p}[f] \equiv f(q, p)$. With abuse of notation Eq. (11) is then generally written as

$$
\frac{\partial}{\partial t} f=\{f, \mathcal{H}\}
$$

That is, one identifies the functional $\mathcal{F}_{q, p}$ with a point in the 'phase space' of this system, i.e. the function $f$ itself. This is completely analogous to the abuse of notation that one commits in writing the canonical equations (10) where the symbols $q$ and $p$ are used to denote both a point in phase space (as arguments of the Hamiltonian function $H$ ) as well as the components of the function expressing the coordinates of that point.

To verify the equivalence between (11) [or (18)] and (1) one has to make use of the boundary conditions $\lim _{q, p \rightarrow \pm \infty} f=0$ and the various definitions we have outlined. It helps to observe that $\delta \mathcal{F}_{q, p}[f ; \delta f]=\delta f$ and from definition (15),

$$
\begin{aligned}
& \frac{\delta \mathcal{F}_{q, p}}{\delta f\left(q^{\prime}, p^{\prime}\right)}=\hat{\delta}\left(q-q^{\prime}\right) \hat{\delta}\left(p-p^{\prime}\right), \\
& \frac{\delta \mathcal{H}}{\delta f}=H
\end{aligned}
$$

where the $\hat{\delta}$ 's are Dirac $\delta$-functions.

\footnotetext{
†Strictly speaking Eq. (16) defines a weak (or a 'Gâteaux') differential, while what is needed is a strong (or 'Fréchet') differential. However, it can be shown that if a strong differential exists it is identical to the weak differential.
} 
Before ending the Section one has to make some important observations concerning the existence of the so-called Casimir functionals $[7,8]$

$$
\mathcal{C}[f]=\int d q d p \chi(f)
$$

where $\chi(f)$ is an arbitrary function. These functionals are all invariant $\{\mathcal{H}, \mathcal{C}\}=0$. The existence of an infinite number of constant of motions has implications in the description of the geometry of the phase space [6]. In the following, however, we will be more interested in the additional property that the Lie-Poisson brackets of a Casimir with any $f$ vanish: $\{f, \mathcal{C}\}=0$. A consequence is that the Morrison Hamiltonian $\mathcal{H}$ is not uniquely defined. Clearly any $\mathcal{H}_{c}=\mathcal{H}+\mathcal{C}$ will generate the same equation of motion as $\mathcal{H}$. As we will see next, this freedom in choosing the Hamiltonian will be crucial to our ability to characterize stability of the linearized motion.

\subsection{Linearized Equation}

To study the solution of the Vlasov equation in the linear approximation we proceed by making a Taylor expansion in (18) around the Haïssinski solution : $f=f_{0}+f_{1}$. Alerted by the observation made in the last paragraph we choose as Hamiltonian $\mathcal{H}_{c}=\mathcal{H}+\mathcal{C}$ with $\mathcal{C}$ to be determined. To carry out the calculation we need a first order expansion around $f=f_{0}$ for the functional derivative $\delta \mathcal{H}_{c} / \delta f[7]$

$$
\left.\frac{\delta \mathcal{H}_{c}}{\delta f} \simeq \frac{\delta \mathcal{H}_{c}}{\delta f}\right|_{f_{0}}+\frac{1}{2} \frac{\delta\left(\delta^{2} \mathcal{H}_{c}\right)}{\delta f_{1}},
$$

where the second variation is defined as $\delta^{2} \mathcal{H}_{c}=\delta^{2} \mathcal{H}_{c}\left[f_{0} ; f_{1}, f_{1}\right]=d /\left.d t\left(\delta \mathcal{H}_{c}\left[f_{0}+t f_{1} ; f_{1}\right]\right)\right|_{t=0}$.

Through first order

$$
\begin{aligned}
\frac{\partial f_{1}}{\partial t} \simeq & \int d q d p f_{1}\left[\hat{\delta}\left(q-q^{\prime}\right) \hat{\delta}\left(p-p^{\prime}\right),\left.\frac{\delta \mathcal{H}_{c}}{\delta f}\right|_{f_{0}}\right]+ \\
& \int d q d p f_{0}\left[\hat{\delta}\left(q-q^{\prime}\right) \hat{\delta}\left(p-p^{\prime}\right), \frac{1}{2} \frac{\delta\left(\delta^{2} \mathcal{H}_{c}\right)}{\delta f_{1}}\right] .
\end{aligned}
$$

Let us now focus our attention on the last term and notice the similarity with the expression defining the Lie-Poisson brackets in (14). The only difference is that here the kernel of the integral is $f_{0}$ as opposed to the dynamical variable $f$. As one can guess the last term in Eq. (23) is yet another instance of Lie-Poisson brackets [7]. Indeed, one can verify that the brackets $\{\cdot, \cdot\}_{L}$ defined by

$$
\{\mathcal{A}, \mathcal{B}\}_{L}[f]=\iint d q d p f_{0}\left[\frac{\delta \mathcal{A}}{\delta f}, \frac{\delta \mathcal{B}}{\delta f}\right]
$$

satisfy all the properties (17). Moreover, observe that if in (23) the term with the derivative $\delta \mathcal{H}_{c} / \delta f$ happened to vanish we could write the linearized equation in a Hamiltonian form

$$
\frac{\partial f_{1}}{\partial t}=\left\{f_{1}, \frac{1}{2} \delta^{2} \mathcal{H}_{c}\right\}_{L}
$$


with the second variation $\delta^{2} \mathcal{H}_{c} / 2$ playing the role of an effective Hamiltonian for the linearized motion. This is where we exploit the freedom of choosing $\mathcal{H}_{c}$ : we will show in a moment that by selecting a suitable Casimir it is indeed possible to make $\mathcal{H}_{c}$ stationary in $f_{0}$ i.e. to have $\delta \mathcal{H}_{c} / \delta f=0$ so as to remove the undesired term in (23).

From definition (21) evaluation of the functional derivative of the Casimir is straightforward $\frac{\delta \mathcal{C}}{\delta f}=\frac{d \chi(f)}{d f}$. Now, we wish to determine a $\chi(f)$ such that

$$
\left.\frac{\delta \mathcal{H}_{c}}{\delta f}\right|_{f_{0}}=\left.\frac{\delta \mathcal{H}}{\delta f}\right|_{f_{0}}+\left.\frac{\delta \mathcal{C}}{\delta f}\right|_{f_{0}}=H_{0}+\left.\frac{d \chi}{d f}\right|_{f_{0}}=0
$$

Being interested in stationary solutions of Haïssinski form $f_{0}=\hat{f}_{0} \exp \left(-H_{0}\right)$ we can immediately conclude that the equation above is satisfied if $d \chi(f) /\left.d f\right|_{f=f_{0}}=\log \left(f_{0} / \hat{f}_{0}\right)$.

Once the equation for the linearized motion has been established we can immediately draw some conclusions regarding stability. First of all, one should observe that the effective Hamiltonian $\delta^{2} \mathcal{H}_{c}$ is an invariant under linear motion (i.e. $\left\{\delta^{2} \mathcal{H}_{c}, \delta^{2} \mathcal{H}_{c}\right\}_{L}=0$, because of the antisymmetry property of $\left.\{\cdot, \cdot\}_{L}\right)$. This suggests that if $\delta^{2} \mathcal{H}_{c}$ or $-\delta^{2} \mathcal{H}_{c}$ were positive definite over the set of all perturbations one could use $\delta^{2} \mathcal{H}_{c}$ itself to define a norm $\ddagger$ i.e. $\left\|f_{1}\right\| \equiv\left|\delta^{2} \mathcal{H}_{c}\left[f_{0} ; f_{1}, f_{1}\right]\right|$. The fact that for all times $t>0$ one has $\left\|f_{1}(t)\right\| \leq\left\|f_{1}(t=0)\right\|$ is a statement of linear stability.

The last task is to evaluate $\delta^{2} \mathcal{H}_{c}=\delta^{2} \mathcal{H}+\delta^{2} \mathcal{C}$ in $f_{0}$. From the expression of the Morrison Hamiltonian (13):

$$
\begin{aligned}
\delta^{2} \mathcal{H} & =\left.\frac{d^{2}}{d t^{2}} \mathcal{H}\left[f_{0}+t f_{1}\right]\right|_{t=0} \\
& =I_{L} \int d q d p f_{1}(q, p) \int d p^{\prime} f_{1}\left(q, p^{\prime}\right),
\end{aligned}
$$

while the second variation of the Casimir functional is

$$
\delta^{2} \mathcal{C}=\left.\frac{d^{2}}{d t^{2}} \int d q d p \int^{f_{0}+t f_{1}} \log \left(\frac{\bar{f}}{\hat{f}_{0}}\right) d \bar{f}\right|_{t=0}=\int d q d p \frac{f_{1}^{2}}{f_{0}} .
$$

In summary,

$$
\frac{1}{2} \delta^{2} \mathcal{H}_{c}=\frac{1}{2} \int d q d p \frac{f_{1}^{2}}{f_{0}}+\frac{I_{L}}{2} \int d q\left[\int f_{1}(q, p) d p\right]^{2}
$$

It is a useful exercise to verify that indeed the reduced equation (25) is identical to the linearized Vlasov equation (7) and that (29) is a linear invariant.

Because the first term on the RHS of (29) is always positive a sufficient condition for linear stability is

$$
\delta^{2} \mathcal{H}_{c}\left[f_{0} ; f_{1}, f_{1}\right]>0, \quad \forall f_{1} \in \Sigma,
$$

where $\Sigma$ is the set of all physically admissible perturbations. The functions $f_{1}$ belonging to $\Sigma$ are required to satisfy the following very mild conditions. They should i) be differentiable,

\footnotetext{
${ }^{\ddagger}$ One has to make sure that this is, in fact, a legitimate definition of a norm - it is in our case.
} 
ii) decay sufficiently fast at infinity in order for the integrals in (29) to exist, and iii) be consistent with charge conservation $\iint f_{1} d q d p=0$.

A simple inspection of the functional (29) shows that condition (30) is always satisfied if $I_{L}>0$. The case $I_{L}<0$ requires some more work and will be discussed in detail in Sec. 5 . We remark that within this framework one cannot prove that (30) is necessary for stability. However, a necessary condition can be established by using the different but closely related argument that will be presented in the next Section.

\section{The Laval-Mercier-Pellat Energy Principle}

Suppose we were presented with an equation of the form

$$
\frac{\partial^{2} f_{1}}{\partial t^{2}}=-\mathcal{L} f_{1}
$$

where $\mathcal{L}$ is a linear operator defined on the space $\Sigma \ni f_{1}$ representing first order perturbations $f_{1}$. If $\mathcal{L}$ were a trivial operator whose action were to multiply the function $f_{1}$ by a constant $L$, i.e. $\mathcal{L} f_{1}=L f_{1}$, then the equation above would simply be that of a harmonic oscillator. Energy conservation would imply stability if the potential energy term $L f^{2} / 2$ were positive for all $f_{1}$ - i.e. if $L>0$. A generalization of this idea to non-trivial operators underlies the Laval-Mercier-Pellat (LMP) energy principle [9, 10]. The key ingredient is the existence of a scalar product $\langle\cdot, \cdot\rangle$ in the space $\Sigma$ with respect to which the operator $\mathcal{L}$ is self-adjoint. It is easy to verify that if such a scalar product exists the 'energy' functional

$$
\mathcal{E}[f]=\mathcal{T}[f]+\mathcal{U}[f]
$$

naturally defined in terms of the 'kinetic energy' $\mathcal{T}[f]=\left\langle f^{\prime}, f^{\prime}\right\rangle / 2$ and 'potential energy' functionals $\mathcal{U}[f]=\langle f, \mathcal{L} f\rangle / 2$ is invariant (i.e. $\mathcal{E}^{\prime}=0$ ) if $f$ is solution of Eq. (31). Here a prime $^{\prime}$ denotes differentiation with respect to time. Because $\mathcal{T}[f]$ is always positive definite if one can prove that the 'potential energy' term $\mathcal{U}[f]$ is positive over all possible displacements $f_{1}$ one can immediately conclude from energy invariance and Eq. (32) that the solution $f$ cannot grow indefinitely (i.e. $\left\langle f^{\prime}, f^{\prime}\right\rangle$ must be bounded). That is, like Eq. (29)

$$
\mathcal{U}\left[f_{1}\right]>0, \quad \forall f_{1} \in \Sigma,
$$

is a sufficient condition for stability. In addition, one can prove that if there exists a displacement $\psi$ such that $\mathcal{U}[\psi]<0$ one can find at least one solution that grows exponentially (see Appendix A). In other terms Eq. (33) unlike Eq. (29) is also necessary for stability.

This is an interesting result but, of course, useful only if one is able to cast the linearized Vlasov equation (7) in the form (31) of a second order equation in time. This can be done using a trick suggested by Antonov $[12,11]$. The idea is to decompose $f$ into even and odd parts with respect to momentum inversion i.e. $f_{1}=f_{+}+f_{-}$with

$$
f_{ \pm}(q, p)=\frac{1}{2}\left[f_{1}(q, p) \pm f_{1}(q,-p)\right] .
$$


If we insert this expression into Eq. (7) and set to zero the even and odd parts separately we find:

$$
\begin{aligned}
& \frac{\partial f_{+}}{\partial t}=\mathcal{D} f_{-}, \\
& \frac{\partial f_{-}}{\partial t}=\mathcal{D} f_{+}+\mathcal{K} f_{+},
\end{aligned}
$$

having used the definitions of the operators $\mathcal{H}$ and $\mathcal{K}$ introduced in Eq. (8) and (9), the facts that $\int_{-\infty}^{\infty} d p f_{-}(q, p)=0$ and $\partial f_{0} / \partial p$ is odd in $p$. After differentiating (36) with respect to time the two equations above can be combined into

$$
\frac{\partial^{2} f_{-}}{\partial t^{2}}=\mathcal{D}^{2} f_{-}+\mathcal{K} \mathcal{D} f_{-} \equiv-\mathcal{L} f_{-}
$$

which is in the form $(31)$, as desired, with $\mathcal{L}=-\left(\mathcal{D}^{2}+\mathcal{K} \mathcal{D}\right)$. The next step is to introduce a scalar product $\langle\cdot, \cdot\rangle$. A suitable choice for any two functions $f, g \in \Sigma$ is

$$
\langle f, g\rangle=\int \frac{f g}{f_{0}} d q d p
$$

with the weigh function $f_{0}$ being the equilibrium distribution.

The key point now is to show that the operator $\mathcal{L}$ is self-adjoint with respect to this scalar product. This can be done, as usual in this kind of calculations, by carrying out integration by parts and using the boundary conditions $\lim _{q, p \rightarrow \pm \infty} f, g=0$. In terms of this scalar product we can then evaluate the 'potential energy' functional

$$
\mathcal{U}\left[f_{-}\right]=\frac{1}{2}\left\langle f_{-}, \mathcal{L} f_{-}\right\rangle=-\frac{1}{2}\left\langle f_{-}, \mathcal{D}^{2} f_{-}\right\rangle-\frac{1}{2}\left\langle f_{-}, \mathcal{K} \mathcal{D} f_{-}\right\rangle .
$$

Again, evaluation of the two terms requires carrying out integrations by parts and use of the boundary conditions:

$$
\begin{aligned}
\left\langle f_{-}, \mathcal{D}^{2} f_{-}\right\rangle & =-\int \frac{\left(\mathcal{D} f_{-}\right)^{2}}{f_{0}} d q d p \\
\left\langle f_{-}, \mathcal{K} \mathcal{D} f_{-}\right\rangle & =-\frac{I_{L}}{2} \int d q\left[\int d p \mathcal{D} f_{-}\right]^{2} .
\end{aligned}
$$

We finally obtain the following expression for the potential energy functional

$$
\mathcal{U}\left[f_{-}\right]=\frac{1}{2} \int \frac{\left(\mathcal{D} f_{-}\right)^{2}}{f_{0}} d p d q+\frac{I_{L}}{2} \int d q\left[\int \mathcal{D} f_{-} d p\right]^{2} .
$$

We recognize that (40) has a form very similar to that of the functional (29). Again, if $I_{L}>0$ then $\mathcal{U}\left[f_{-}\right]$is certainly positive for all $f_{-} \in \Sigma_{-}$implying that the system is linearly stable [recall (33)]. Here, with $\Sigma_{-}$we indicate the subset of the physically admissible perturbations belonging to $\Sigma$ that are invariant under momentum inversion. On the ohter hand, if $I_{L}<0$ and $\mathcal{U}\left[f_{-}\right]<0$ for some displacement $f_{-}$the motion is unstable. 


\section{Discussion of Stability}

We have already observed that the condition (30) for stability, i.e. $\delta^{2} \mathcal{H}_{c}>0$, is immediately satisfied if $I_{L}>0$. For $I_{L}<0$ the requirement $\delta^{2} \mathcal{H}_{c}>0$ can be restated as

$$
\left|I_{L}\right|<\frac{\int f^{2} / f_{0} d q d p}{\int d q\left(\int f d p\right)^{2}} \equiv \mathcal{M}[f]
$$

for all $f \in \Sigma$. The last equation defines the functional $\mathcal{M}[f]$. Clearly, the minimum of the functional $\mathcal{M}[f]$

$$
I_{L}^{u p}=-\min _{f \in \Sigma} \mathcal{M}[f]
$$

yields a upper bound $I_{L}^{u p}$ to the threshold of stability. That is, the motion is stable if $I_{L}>I_{L}^{u p}$.

The task now is to determine $I_{L}^{u p}$ by minimizing $\mathcal{M}[f]$. To this end it is convenient to introduce a 'change of variable' in the definition of $\mathcal{M}[f]$ by re-scaling the trial functions with respect to the Haïssinski solution $f_{0}$ and write $f=\tilde{f} \sqrt{f_{0}}$. With the understanding that $\mathcal{M}$ is now defined in terms of the functions $\tilde{f}$ :

$$
\mathcal{M}[\tilde{f}]=\frac{\int \tilde{f}^{2} d q d p}{\int d q\left(\int \tilde{f} \sqrt{f_{0}} d p\right)^{2}}=\frac{\sqrt{2 \pi} \int \tilde{f}^{2} d q d p}{\int d q \rho_{0}(q)\left(\int \tilde{f} e^{-p^{2} / 4} d p\right)^{2}}
$$

where in the second equality we have used the form (5) of the Haïssinski solution. Next, we invoke the Schwartz inequality

$$
\left(\int \tilde{f} e^{-p^{2} / 4} d p\right)^{2} \leq \int \tilde{f}^{2} d p \int e^{-p^{2} / 2} d p
$$

and write

$$
\mathcal{M}[\tilde{f}] \geq \frac{\int \tilde{f}^{2} d q d p}{\int d q \rho_{0} \int \tilde{f}^{2} d p}=\frac{\int \tilde{f}^{2} d q d p}{\int \rho_{0} \tilde{f}^{2} d q d p} .
$$

Because Haïssinski solutions $\rho_{0}(q)$ at $q=0$ have a maximum $\hat{\rho}_{0}$ (see remark at the end of this Section), it is clear that the right hand side of (44) is always larger than $1 / \hat{\rho}_{0}$. One can verify that $1 / \hat{\rho}_{0}$ is in fact a minimum for the operator $\mathcal{M}$ and not just a lower bound by considering the family of functions $\tilde{f}_{\lambda}=e^{-p^{2} / 4} h_{\lambda}(q)$, where $h_{\lambda}(q)=e^{-q^{2} / 2 \lambda} / \sqrt{2 \pi \lambda}-A_{\lambda} /[1+$ $\left.(\lambda q)^{2}\right]$ with $A_{\lambda}$ being a normalization constant chosen to guarantee that $\iint \tilde{f}_{\lambda} \sqrt{f_{0}} d q d p=0$ so that $\tilde{f}_{\lambda} \sqrt{f_{0}} \in \Sigma$. It is easy to see that in the limit $\lambda \rightarrow 0$ we have $\mathcal{M}\left[\tilde{f}_{\lambda}\right] \rightarrow 1 / \hat{\rho}_{0}$. Therefore, we conclude that $I_{L}^{u p}=1 / \hat{\rho}_{0}$.

Similarly, by considering the expression (40) for the functional $\mathcal{U}$ we recognize that condition (33) when $I_{L}<0$ is equivalent to

$$
\left|I_{L}\right|<\frac{\int\left(\mathcal{D} f_{-}\right)^{2} / f_{0} d q d p}{\int d q\left(\int \mathcal{D} f_{-} d p\right)^{2}}=\mathcal{M}\left[\mathcal{D} f_{-}\right] .
$$

Notice that one cannot infer that $\min _{f_{-} \in \Sigma_{-}} \mathcal{M}\left[\mathcal{D} f_{-}\right]=\min _{f \in \Sigma} \mathcal{M}[f]$ simply on the basis of the similarity between (41) and (45) because the codomain $\mathcal{D} \Sigma_{-}$is only a subset of $\Sigma$. 
However, the same conclusion can be reached by repeating the argument above with slight modifications. As before, we do the scaling $f_{-}=\tilde{f}_{-} \sqrt{f_{0}}$. Because $\mathcal{D} f_{0}=\left[H_{0}, f_{0}\left(H_{0}\right)\right]=0$ it is $\mathcal{D}\left(f_{-} \sqrt{f_{0}}\right)=\sqrt{f_{0}} \mathcal{D} f_{-}$enabling us to write

$$
\begin{aligned}
\mathcal{M}\left[\mathcal{D} \tilde{f}_{-}\right] & =\frac{\sqrt{2 \pi} \int\left(\mathcal{D} \tilde{f}_{-}\right)^{2} d q d p}{\int d q \rho_{0}\left(\int e^{-p^{2} / 4} \mathcal{D} \tilde{f}_{-} d p\right)^{2}} \\
& \geq \frac{\int\left(\mathcal{D} \tilde{f}_{-}\right)^{2} d q d p}{\int \rho_{0}\left(\mathcal{D} \tilde{f}_{-}\right)^{2} d q d p}
\end{aligned}
$$

where, again, we have made use of the Schwartz inequality. The last term on the right is $\geq$ $1 / \hat{\rho}_{0}$. In fact, one can verify that $1 / \hat{\rho}_{0}$ is a minimum by evaluating the limit $\lim _{\lambda \rightarrow 0} \mathcal{M}\left[\mathcal{D} \tilde{f}_{-}^{\lambda}\right]=$ $1 / \hat{\rho}_{0}$ over the family of functions $\tilde{f}_{-}^{\lambda}=h(q) p e^{-p^{2} / 4} /\left(p^{2}+\lambda\right)$, with $h(q)=q e^{-q^{2} / 2 \lambda} / \sqrt{2 \lambda}$. Therefore, $\min _{f_{-} \in \Sigma_{-}} \mathcal{M}\left[\mathcal{D} \tilde{f}_{-}\right]=1 / \hat{\rho}_{0}$.

Because (45) is a necessary condition for stability, we conclude that

$$
I_{L}^{c r i t}=-\frac{1}{\hat{\rho}_{0}}
$$

is in fact a critical current, that is the motion is stable if and only if $I_{L}>I_{L}^{c r i t}$.

This conclusion applies to bunches as well to coasting beams. However, for bunched beams we encounter the peculiar situation that the stability condition is the same as the condition for the existence of the equilibrium (Haïssinski) solution. This can be easily seen by inspection of the Haïssinski equation (6). Firstly, one observes that in order for physically acceptable solutions to exists it must be $\rho_{0}(q) I_{L}+1>0$ for all $q$. Indeed, if this quantity were negative at one point the solution would not be normalizable. Secondly, the derivative $d \rho_{0} / d q$ of a normalizable solution is always negative for $q>0$ and positive for $q<0$. In other terms, $\hat{\rho}_{0}=\rho_{0}(q=0)$ is the function maximum and the condition for a physical solution to exist can be restated as $\hat{\rho}_{0} I_{L}+1>0$, consistent with (47). We conclude that in the presence of a purely inductive impedance for bunched beams - both above and below transition - a Haïssinski solution, if it exists, is always stable. For negative $I_{L}$ with $I_{L}<I_{L}^{\text {crit }}$ an equilibrium solution, however, does not exist and a linear motion analysis does not apply. Obviously, in this case it is still reasonable to interpret the motion as unstable.

For coasting beams $\hat{\rho}_{0}=1 / 2 \pi$ (see end of Sec. 2) so that $I_{L}^{\text {crit }}=-2 \pi$. This is identical to the threshold predicted by the standard (Landau) linear theory based on wave expansion (see e.g. [15]). According to this theory one looks for solutions in the form $f_{1} \propto e^{-i \omega t+i n q}$ and then determines the emergence of a positive imaginary part in the frequency $\omega$ with the help of the dispersion relation $1+\frac{I_{L}}{2 \pi}[1+i \sqrt{\pi} \Omega w(\Omega)]=0$, where $w$ is the error function of complex argument and $\Omega=\omega / \sqrt{2} n$. The stability condition one deduces is $I_{L} / 2 \pi>-1$, in accordance with our analysis. 


\section{Conclusions}

The main result of this paper is a demonstration that longitudinal bunch distributions in the form of Haïssinski equilibria - if they exist - are always stable in the presence of a purely inductive impedance. This is the case whether the potential well distortion associated with the impedance causes bunch lengthening or shortening.

This result should be contrasted to the standard analysis based on mode decomposition. Using this method the frequencies $\omega^{2}$ for the normal modes $\propto \exp (-i \omega t)$ are determined as eigenvalues of an infinite-dimension matrix. For a purely inductive impedance the most one can do without getting into a numerical calculation is to show that such a matrix is symmetric implying that $\omega^{2}$ is real. This is consistent with $\omega$ being either real (implying stability) or purely imaginary (implying instability) [4]. Incidentally, that for normal modes $\omega^{2}$ must be real can also be quickly established from Eq. (31).

We conclude with three final remarks. First, we mention that results similar to those highlighted in this paper could also have been derived by considering more general nonHaiissinki equilibria in the form of strictly decreasing monotonic functions of the singleparticle Hamiltonian.

Next, it is worth noticing that the threshold (47) could have been obtained by invoking the Boussard criterion [16]. Boussard stated that if the wavelength of the instability is small compared to the bunch length one is permitted to apply the linear analysis valid for coasting beams. The current of the equivalent coasting beam should be the same as the peak current for the bunched beam. The example discussed in this paper is an interesting instance in which Boussard's criterion appears to give the exact answer. We recall that Boussard formulated his statement purely on the basis of physical intuition - although later there have been attempts to put it on a more rigorous ground [17].

Finally, we would like to follow up on the observation made in the Introduction regarding the exclusive applicability of the methods discussed in this paper to purely inductive wakes. The problem with more general wake-fields is the lack of symmetry in the interchange between $q$ and $q^{\prime}$ in the primitive of the wake field function $S\left(q-q^{\prime}\right)=\int^{q-q^{\prime}} W(\tau) d \tau$. In the case of the LMP method of Sec. 4 this prevents the operator $\mathcal{L}$ of Eq.(31) from being self-adjoint. As for the Hamiltonian method of Sec. 3 the difficulty is that the functional derivative of the Morrison functional Hamiltonian (13) does not equal the single-particle Hamiltonian [that is, Eq. (20) does not hold]. Whether a different choice of the functional Hamiltonian could work in the more general case is not clear at this point.

\section{Acknowledgments}

I am grateful to R. Ruth, A. Chao, and in particular R. Warnock for their comments. Work supported by DOE contract DE-AC03-76SF0051. 


\section{Appendix: Necessity of the Laval-Mercier-Pellat Con- dition}

Here we report the proof [10] that condition (33), i.e. $\mathcal{U}[f]>0, \quad \forall f \in \Sigma$ is necessary for instability. In particular it is shown that the functional $\mathcal{I}[f]=\langle f, f\rangle$-representing a norm of the displacement $f$ - grows at least exponentially if there exists a displacement $\psi$ such that $\mathcal{U}[\psi]<0$. The reasoning goes as follows. We take such an $\psi$ to be the initial condition $f_{+}(t=0)=\psi$ and we choose the initial time-derivative to be $f_{+}^{\prime}(t=0)=p_{0} \psi$ where $p_{0}$ is a positive number defined as $p_{0}=[-\mathcal{U}[\psi] / \mathcal{I}[\psi]]^{\frac{1}{2}}$. First, observe that with this choice the energy functional is initially zero

$$
\begin{aligned}
\mathcal{E}\left[f_{+}(t=0)\right] & =\frac{1}{2}\left\langle p_{0} \psi, p_{0} \psi\right\rangle+\frac{1}{2}\langle\psi, \mathcal{L} \psi\rangle \\
& =\frac{1}{2}\left(p_{0}^{2} \mathcal{I}[\psi]+\mathcal{U}[\psi]\right)=0
\end{aligned}
$$

and because the energy functional is invariant it remains zero at later times.

From the definition of $\mathcal{I}[f]$ if we take the first derivative with respect to time we find $\mathcal{I}^{\prime}=2\left\langle f^{\prime}, f\right\rangle$. As a result of the Schwartz inequality, $\langle f, g\rangle^{2} \leq\langle f, f\rangle\langle g, g\rangle$ we can write the estimate

$$
\left(\mathcal{I}^{\prime}[f]\right)^{2}=4\left\langle f, f^{\prime}\right\rangle^{2} \leq 4\langle f, f\rangle\left\langle f^{\prime}, f^{\prime}\right\rangle=8 \mathcal{T}[f] \mathcal{I}[f]
$$

On the other hand by taking the second derivative of $\mathcal{I}$ we have:

$$
\mathcal{I}^{\prime \prime}[f]=4 \mathcal{T}[f]-4 \mathcal{U}[f]=8 \mathcal{T}[f]
$$

where the last equation follows from the energy $\mathcal{E}=\mathcal{T}+\mathcal{U}$ vanishing. Next, we combine (49) and (50) to obtain

$$
\frac{\mathcal{I}^{\prime 2}}{\mathcal{I}} \leq \mathcal{I}^{\prime \prime} \quad \text { or } \quad \frac{\mathcal{I}^{\prime \prime}}{\mathcal{I}}-\frac{\mathcal{I}^{\prime 2}}{\mathcal{I}^{2}}=\frac{d}{d t}\left(\frac{\mathcal{I}^{\prime}}{\mathcal{I}}\right) \geq 0
$$

from which we infer that

$$
\frac{\mathcal{I}^{\prime}}{\mathcal{I}} \geq \text { const }
$$

The constant of integration is determined by the initial condition const $=2 p_{0}$, (at $t=0$ we have $\mathcal{I}^{\prime}=2\left\langle p_{0} \psi, \psi\right\rangle$, that is $\left.\mathcal{I}^{\prime} / \mathcal{I}=2 p_{0}\right)$. We finally conclude that $\mathcal{I}^{\prime} \geq 2 p_{0} \mathcal{I}$ or $\mathcal{I} \geq \mathcal{I}_{0} \exp \left(2 p_{0} t\right)$

\section{References}

[1] L. Landau, J. Phys. USSR 10, 25 (1946).

[2] N. Van Kampen, Physica 21, 949 (1955).

[3] K. Case, Annals of Phys. 7, 349 (1959). 
[4] K. Oide, Part. Acc. 51, 43 (1995). Also as KEK Preprint 94-138, November 1994.

[5] An attempt to improve on this aspect of the mode decomposition method will be discussed in a paper by R. Warnock, M. Venturini, and J. Ellison, to appear soon.

[6] P. Morrison, Phys. Lett. A 80, 383 (1980); see P. Morrison, Rev. of Mod. Phys. 70, 467 (1998) for an introduction to non-canonical methods.

[7] D. Holm, J. Marsden, T. Ratiu, and A. Weinstein, Phys. Reports, 123, 1 (1985).

[8] H. Kandrup, Astrophysical Journ. 351, 104 (1990).

[9] G. Laval, C. Mercier, and R. Pellat, Nuclear Fusion, 5, 156 (1965).

[10] R. Kulsrud and J. Mark, Astrophysical Journ. 160, 471 (1970).

[11] J. Binnet and S. Tremaine, Galactic Dynamics, Princeton University Press, Princeton (1987).

[12] V.A. Antonov, Astr. Zh., 37, 918 (1960) (translated in Sov. Astron. 4, 859).

[13] R. Warnock and J. Ellison, A General Method of Propagation of Phase Space Distribution with Application to the Sawtooth Instability, 2nd ICFA Advanced Accelerator Workshop on the Physics of High Brightness Beams, UCLA 9-12 Nov. 1999, World Scientific, Singapore (2000).

[14] A. Chao, Physics of Collective Instabilities in High Energy Accelerators, John Wiley \& Son, New York (1993).

[15] S.Y. Lee, Accelerator Physics, World Scientific, Singapore (1999).

[16] D. Boussard, CERN LABII/RF/INT/75-2 (1975).

[17] J.M. Wang and C. Pellegrini, On the Condition for a Single Bunch High Frequency Fast Blow-Up, XI Int. Conf. on High Energy Accelerators, Geneva CH, July 7-11, 1980, Proceedings. 